\title{
Tau mass measurement at BES-III
}

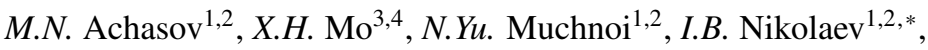 \\ S.A. Privalov ${ }^{1,2}$, and J.Y. Zhang ${ }^{3}$ on behalf of the BES-III collaboration \\ ${ }^{1}$ Budker Institute of Nuclear Physics, Novosibirsk, Russia \\ ${ }^{2}$ Novosibirsk State University, Novosibirsk, Russia \\ ${ }^{3}$ Institute of High Energy Physics, Beijing, China \\ ${ }^{4}$ University of Chinese Academy of Sciences, Beijing, China
}

\begin{abstract}
A scan of the threshold region of the process $e^{+} e^{-} \rightarrow \tau^{+} \tau^{-}$with integrated luminosity about $140 \mathrm{pb}^{-1}$ was performed with the BES-III detector at the BEPC-II collider in order to measure the $\tau$-lepton mass. The beam energy was determined by the Compton backscattering method. To verify the measurement accuracy scans of the $J / \psi$ and $\psi^{\prime}$ resonances were performed.
\end{abstract}

\section{Introduction}

The $\tau$-lepton mass is a Standard Model parameter, which should be determined with high accuracy. The most precise method of mass determination is to extract its value from the energy dependence of the $e^{+} e^{-} \rightarrow \tau^{+} \tau^{-}$cross section near the reaction threshold. This measurement is an important part of the physical program of the BES-III experiment [1] at the BEPC-II collider.

The threshold scan for the $\tau$-lepton mass measurement requires precise determination of the beam energy. The beam energy of the BEPC-II collider is measured by the beam energy measurement system (BEMS) based on the Compton backscattering technique [2]. The $\tau$ lepton mass measurement was performed in 2011 with integrated luminosity of $24 \mathrm{pb}^{-1}$ [3]. The statistical accuracy of the measurement was about $120 \mathrm{keV}$ limited by experimental statistics, while the uncertainty due to the beam energy determination was about $40 \mathrm{keV}$. Therefore a new experiment with integrated luminosity of $140 \mathrm{pb}^{-1}$ was performed in 2018 . The statistical accuracy of this measurement is expected to be about $50 \mathrm{keV}$.

\section{Beam energy determination}

The Compton backscattering technique is based on determination of the maximal energy of the scattered photons $\omega_{\max }$, which is related to the electron energy $E$ by the kinematics of the Compton scattering. If one measures $\omega_{\max }$, then the electron energy can be calculated:

$$
E=\frac{\omega_{\max }}{2}\left(1+\sqrt{1+\frac{m_{e}^{2}}{\omega_{0} \omega_{\max }}}\right),
$$

\footnotetext{
*e-mail: I.B.Nikolaev@inp.nsk.su
} 
where $m_{e}$ is the electron mass, $\omega_{0}$ is the initial photon energy ${ }^{1}$. The BEMS layout is shown in Fig. 1. The system consists of the $\mathrm{CO}_{2}$ laser with a wavelength $\lambda=10.591035 \mu \mathrm{m}\left(\omega_{0} \approx\right.$ $0.117 \mathrm{eV}$ ), optical and laser-to-vacuum insertion system to transport the laser beam to the interaction region where the laser beam collides with electron or positron beams, and the High Purity Germanium (HPGe) detector to measure spectra of backscattering photons. Its energy scale is calibrated by using well-known sources of $\gamma$-radiation and by a precise pulse generator [4].

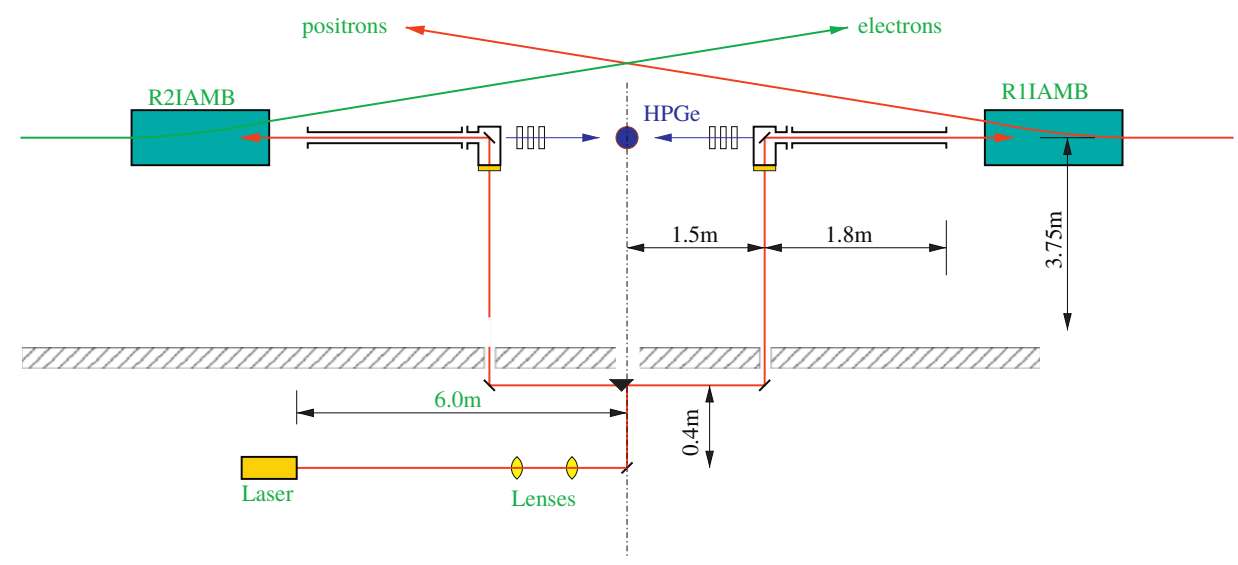

Figure 1. Beam Energy Measurement System [2].

In 2017, before the $\tau$-mass scan experiment, the BEMS accuracy was studied by comparison of the well-known mass of the $\psi^{\prime}$ resonance with its value obtained using the system. The bias of the center-of-mass energy obtained using BEMS with respect to the true value was $52 \pm 42 \mathrm{keV}$.

\section{Experiment}

Data taking in 2018 included the following steps:

1. scan of $J / \psi$ resonance. Data was collected at eight energy points with a total integrated luminosity of about $30 \mathrm{pb}^{-1}$,

2. scan of the $e^{+} e^{-} \rightarrow \tau^{+} \tau^{-}$reaction threshold. Data was collected at five energy points with a total integrated luminosity of about $140 \mathrm{pb}^{-1}$,

3. scan of $\psi^{\prime}$ resonance. Data was collected at ten energy points with a total integrated luminosity of about $70 \mathrm{pb}^{-1}$.

The data collected at the $\psi$ resonances is used to verify the accuracy of the beam energy determination. The BEPC-II energy spread $\sigma_{\mathrm{W}}$ is also determined from the scans. For the $\tau$-threshold scan the five-point scenario proposed in Ref. [5] was used. The data at point 1 was collected below the $e^{+} e^{-} \rightarrow \tau^{+} \tau^{-}$threshold to measure the background. The highest sensitivity to the tau mass is achieved by taking data exactly at the threshold. This statistics was

\footnotetext{
${ }^{1}$ Throughout the paper the natural system of units $\hbar=1$ and $c=1$ is used
} 
shared between two points 2 and 3 in order to take into account the $\tau$-lepton mass uncertainty. The point 4 is used for the $\chi^{2}$ test of the $\tau^{+} \tau^{-}$production curve. The point 5 is intended for the determination of the $\tau^{+} \tau^{-}$detection efficiency.

\section{Data analysis}

The integrated luminosity is determined using $e^{+} e^{-} \rightarrow \gamma \gamma$ events:

$$
L=N^{\gamma \gamma} / \sigma^{\gamma \gamma}
$$

where $N^{\gamma \gamma}$ is the number of selected events and $\sigma^{\gamma \gamma}$ is the visible cross section of the process $e^{+} e^{-} \rightarrow \gamma \gamma$. In order to obtain $N^{\gamma \gamma}$, events were selected with two opposite neutral tracks and the energy deposition in the barrel calorimeter close to the beam energy and without charged tracks coming from the interaction point.

The masses of the $J / \psi$ and $\psi^{\prime}$ mesons are measured using events of $e^{+} e^{-} \rightarrow$ hadrons. Events with at least three charged tracks coming from the interaction point were used. In order to have good momentum measurement and suppress Bhabha background, these tracks should hit the barrel calorimeter. The beam background was suppressed using requirements on the energy deposition in the calorimeter and transverse momentum of the tracks. An additional requirement on the value of sphericity

$$
S=\frac{3}{2}\left(\lambda_{2}+\lambda_{3}\right)
$$

was imposed. Here $\lambda_{1} \geq \lambda_{2} \geq \lambda_{3}$ are eigenvalues of the sphericity tensor:

$$
S^{i j}=\left(\sum_{n=1}^{N_{q}} p_{n}^{i} p_{n}^{j}\right) /\left(\sum_{n=1}^{N_{q}}\left|\mathbf{p}_{n}\right|^{2}\right), \quad i, j=1,2,3
$$

The preliminary results of the $\psi$ resonance mass measurements are shown in Fig. 2. Comparison of these results with the PDG values of the resonance masses allows us to obtain the correction to the center-of-mass energy measured by BEMS $\Delta W=-78 \pm 28 \mathrm{keV}$ and the beam energy spread at the tau threshold $\sigma_{\mathrm{W}}=1.24 \pm 0.01 \mathrm{MeV}$.
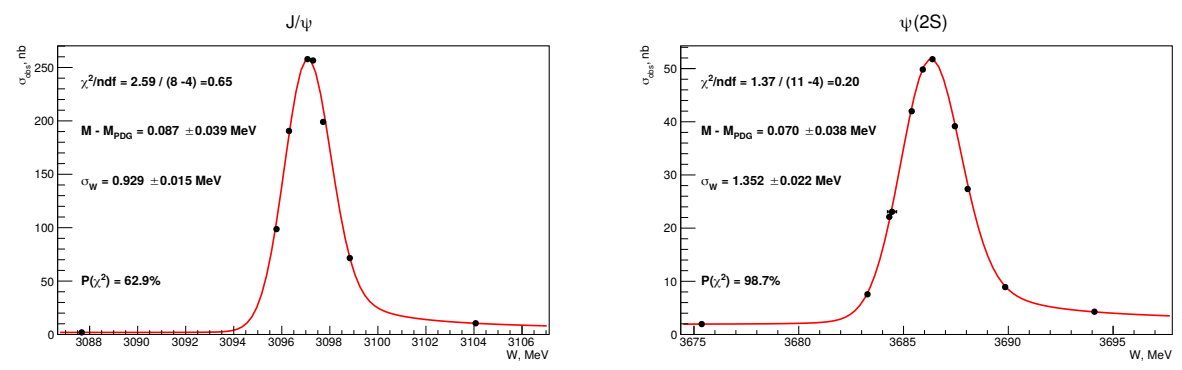

Figure 2. The result of scans of $\psi$ resonances.

Analysis of the process $e^{+} e^{-} \rightarrow \tau^{+} \tau^{-}$is based on $\tau$ pair decays to $e \mu, e \pi, e \rho, \mu \pi, \mu \rho, \pi \rho$, $e e, \mu \mu, \pi \pi$. Thus events with two oppositely charged tracks originated from the interaction point were selected. The tracks must have $|\cos \theta|<0.93$ to get good momentum measurement in the drift chamber. The $d E / d x$, time-of-flight and muon systems were used for particle identification. 
Events do not containing a $\rho$ meson should not have photons with the energy deposition above $25 \mathrm{MeV}$. Events which contain the $\rho$-meson were selected with the requirement of two photons with energy deposition larger than $50 \mathrm{MeV}$ or $25 \mathrm{MeV}$ in the endcap or barrel calorimeters, respectively. The invariant mass of these neutral tracks was required to be close to the $\pi^{0}$ mass, and the invariant mass of two photons and the particle identified as a pion should be close to the $\rho$-meson mass.

The beam background was suppressed by requirements on the energy deposition in the calorimeter and transverse momentum. The background from the ISR processes was rejected by restrictions on a polar angle of a missing photon and on the invariant mass of the charged tracks. The background from the $e^{+} e^{-} \rightarrow e^{+} e^{-} \gamma^{*} \gamma^{*} \rightarrow e^{+} e^{-} X$ processes was suppressed by a requirement on the ratio of the transverse momentum to the missing energy (PTEM):

$$
P T E M=P_{\mathrm{T}} / E_{\mathrm{mis}}=\frac{\left|\vec{p}_{1 \mathrm{t}}+\vec{p}_{2 \mathrm{t}}\right|}{W_{\mathrm{cm}}-\left|p_{1}\right|-\left|p_{2}\right|}
$$

About 2400 events were selected. The visible cross section was fit by using the maximum likelihood method. The function to be minimized was

$$
\chi^{2}=-2 \sum_{i}\left[N_{i}^{\exp }-N_{i}+N_{i} \ln \frac{N_{i}}{N_{i}^{\exp }}\right]
$$

where $N_{i}^{\text {exp }}$ and $N_{i}$ are the expected and selected numbers of events for point $i$ :

$$
N_{i}^{\exp }=L_{i}^{\gamma \gamma}\left(\varepsilon \cdot \epsilon_{i}^{\mathrm{cor}} \cdot \sigma_{\tau \tau}\left(W_{i}, \sigma_{\mathrm{W}}, M_{\tau}\right)+\sigma_{\mathrm{BG}}\right),
$$

where $L_{i}$ is the integrated luminosity, $\sigma_{\mathrm{BG}}$ background cross section, $\varepsilon$ is the $\tau$-pair detection efficiency, $\epsilon_{i}^{\text {cor }}$ is the correction to the detection efficiency determined from Monte Carlo simulation, $\sigma_{\mathrm{W}}$ is the center-of-mass energy spread and $\sigma_{\tau \tau}$ is the tau production cross section:

$$
\sigma_{\tau \tau}\left(W, \sigma_{\mathrm{W}}, M_{\tau}\right)=\int_{2 M_{\tau}}^{\infty} \frac{\exp \left(-\frac{\left(W-W^{\prime}\right)^{2}}{2 \sigma_{W}^{2}}\right) d W^{\prime}}{\sqrt{2 \pi} \sigma_{W}} \int_{0}^{1-4 \frac{M_{\tau}^{2}}{W^{\prime 2}}} \sigma_{f}\left(W^{\prime 2}(1-x)\right) F\left(x, W^{\prime 2}\right) d x
$$

with $F\left(x, s^{\prime}\right)$ is the ISR correction kernel;

$$
\sigma_{f}(s)=\frac{4 \pi \alpha^{2}}{3 s} \frac{v\left(3-v^{2}\right)}{2} \frac{F_{\mathrm{r}}(v) F_{\mathrm{c}}(v)}{\left|1-\Pi\left(s, M_{\tau}\right)\right|^{2}}, \quad v=\sqrt{1-\frac{4 M_{\tau}^{2}}{s}},
$$

where $\Pi\left(s, M_{\tau}\right)$ is the vacuum polarization operator, $F_{\mathrm{r}}(v)$ is the final-state radiative and relativistic correction [6] and $F_{\mathrm{c}}(v)$ is the correction for the final-state Coulomb interaction. The statistical accuracy of the fit is around $50 \mathrm{keV}$. The analysis is in progress.

\section{Conclusion}

In 2018 the $\tau$-lepton threshold scan for the new $\tau$-lepton mass measurement was performed with integrated luminosity about $140 \mathrm{pb}^{-1}$ with the BES-III detector. The Beam Energy Measurement System was calibrated by using scans of the $J / \psi$ and $\psi^{\prime}$ resonances. The correction to the BEMS center-of-mass energy scale $-78 \pm 28 \mathrm{keV}$ was obtained. The analysis of the $e^{+} e^{-} \rightarrow \tau^{+} \tau^{-}$process is in progress. 


\section{Acknowledgments}

The work was supported by the Russian Foundation for Basic Research (RFBR): grant 1852-53014; National Natural Science Foundation of China (NSFC): 11811530073, 11375206; Major State Basic Research Development Program: 2015CB856700, 2015 CB856705.

\section{References}

[1] M. Ablikim et al., Nucl. Instr. Meth. A 614, 345 (2010)

[2] E.V. Abakumova et al., Nucl. Instr. Meth. A 659, 21 (2011)

[3] M. Ablikim et al., Phys. Rev. D 90 (2014)

[4] M.N. Achasov, N.Yu. Muchnoi, JINST 12, C08007 (2017)

[5] M.N. Achasov et al., Chin.Phys. C 36, 573 (2012)

[6] M.B. Voloshin, Phys. Lett. B 556, 153 (2003), hep-ph/0212207 